\title{
Estabilização da Placa Aterosclerótica por Stents Farmacológicos e Não-Farmacológicos em Modelo Experimental de Fibroateroma de Capa Fina em Coelhos
}

\author{
Dario Echeverri', K-Raman Purushothaman², Deborah Kilpatrick³ \\ William N. O'Connor ${ }^{4}$, Pedro R. Moreno ${ }^{5}$
}

\section{RESUMO}

Introdução: Stents farmacológicos e stents não-farmacológicos (SNF) são utilizados no tratamento de placas ateroscleróticas instáveis e podem levar à estabilização dos fibroateromas de capa fina (FACF). Este estudo foi desenhado para avaliar os efeitos estabilizadores dos SNF e dos stents farmacológicos em modelo experimental de FACF. Método: O estudo avaliou 16 coelhos hipercolesterolêmicos da raça Nova Zelândia, acompanhados por quatro anos, dos quais 6 receberam SNF, 5 receberam stents com liberação de everolimus (SLE) e 5, stents com liberação de 17-b estradiol (SLB) (Guidant - Santa Clara, Califórnia, Estados Unidos). Um stent com polímero também foi implantado em cada animal. Análises histológicas aos 28 dias dos FACF não tratados vs. FACF com implante de SLE, SLB e SNF foram realizadas. Resultados: Os FACF tratados com SNF, SLE e SLB mostraram redução da área lipídica de $62 \%, 67 \%$ e $61 \%$ e aumento da espessura da capa de $188 \%, 98 \%$ e $140 \%$, respectivamente $(p<0,0001$ para todos). Ruptura dos FACF induzida pela haste foi encontrada em $63 \%$ das seções histológicas que continham o stent, associada a espessamento da neoíntima nos SNF $(p=0,03)$, mas não nos SLE ou nos SLB $(p=n s)$. Conclusões: $O$ tratamento dos FACF com SNF, SLE e SLB reduz o acúmulo de lípides e aumenta a espessura da capa fibrosa. A ruptura da capa fibrosa

\section{SUMMARY}

\section{Plaque Stabilization by Bare Metal and Drug-Eluting Stents in an Experimental Rabbit Model of Thin Cap Fibroatheroma}

Background: Bare metal stents (BMS) and drug-eluting stents (DES) are used to treat unstable plaques and may stabilize thin cap fibroatheromas (TCFA). This study was designed to evaluate stabilizing effects of bare compared to Everolimus (EES) and Beta-Estradiol (BES) eluting stents in a chronic atherosclerotic experimental animal model of TCFA. Methods: Sixteen New Zealand hypercholesterolemic rabbits followed for 4 years were studied. Six animals received BMS, 5 EES and 5 BES (Guidant - Santa Clara, CA, USA). One polymer stent per animal was also implanted. Histologic analysis at 28 days of de-novo vs. BMS, EES, and BES stented TCFA were performed. Results: BMS, EES, and BES stented TCFA showed reductions in lipid area by $62 \%, 67 \%$, and $61 \%$, and increases in cap thickness by $188 \%, 98 \%$, and $140 \%$ respectively ( $p<0.0001$ for all). Strut-induced ruptured TCFA was found in $63 \%$ of stented sections and was associated with increased neointima in BMS $(p=0.03)$ but not in EES or BES $(p=n s)$. Conclusions: Stenting thin cap fibroatheroma with BMS, EES and BES reduces lipid accumulation and increases cap thickness. Strut-induced fibrous cap rupture was frequently found

\footnotetext{
Servicio de Hemodinamia e Intervencionismo Cardiovascular, Laboratorio de Investigación en Función Vascular, Facultad de Medicina, Universidad del Rosario - Fundación Cardiolnfantil Instituto de Cardiología - Bogotá, Colômbia.

2 Cardiovascular Biology Research Lab. Mount Sinai School of Medicine - New York, NY, Estados Unidos.

3 Research and Technology Guidant Corporation CEISMC - Atlanta, GA, Estados Unidos.

${ }^{4}$ University of Kentucky - Lexington, KY, Estados Unidos.

5 Mount Sinai Medical Center - New York, NY, Estados Unidos.

Suporte Financeiro: Guidant Corporation - Santa Clara, CA, Estados Unidos.

Correspondência: Dario Echeverri. Calle 163 A número 13B - 60 Primer Piso - Bogotá, Colombia - Tel.: (57-1) 679-1192/(57-1) 667-2727 - Ext.: 1114/4322 - Fax: (57-1) 669-0382

E-mail: decheverri@cardioinfantil.org, funcionvascular@cardioinfantil.org Recebido em: 28/3/2008 • Aceito em: 7/5/2008
} 
Echeverri D, et al. Estabilização da Placa Aterosclerótica por Stents Farmacológicos e Não-Farmacológicos em Modelo Experimental de Fibroateroma de Capa Fina em Coelhos. Rev Bras Cardiol Invas. 2008;16(2):170-177.

induzida pela haste do stent foi achado freqüente e foi associada a maior formação de neo-íntima nos SNF, mas não nos SLE e SLB.

DESCRITORES: Aterosclerose. Contenedores. Reestenose coronária. Recidiva. Modelos animais. Coelhos.

$\mathrm{T}$ erapias de sucesso para estabilizar placas ateroscleróticas vulneráveis são necessárias para prevenir as complicações trombóticas da ruptura da placa. O processo inflamatório generalizado e lesões coronárias multifocais destacam a importância da terapia sistêmica como forma ideal de prevenção $0^{1,2}$. Todavia, uma terapia farmacológica agressiva, redutora de lípides e antitrombótica pode ser insuficiente para alcançar essa meta, já que reduz somente $25 \%$ a $31 \%$ dos eventos coronários. Embora as terapias farmacológicas de estabilização sistêmica das placas sejam uma boa alternativa, muitos eventos continuam a ocorrer em pacientes que recebem a melhor terapia atualmente disponível ${ }^{3}$.

Por outro lado, apesar da forte evidência de inflamação sistêmica e de lesões multifocais na árvore coronária, mais de $60 \%$ dos pacientes com síndromes isquêmicas agudas apresentam doença em um vaso e mais de $80 \%$, em dois vasos ${ }^{4}$. Conseqüentemente, a maioria dos pacientes apresenta doença focal, ao invés de doença difusa, na ocasião da manifestação de seu quadro coronário. Essa distribuição focal da aterosclerose coronária pode explicar em parte a superioridade da angioplastia coronária transluminal percutânea quando comparada à trombólise sistêmica no tratamento de infarto agudo do miocárdio ${ }^{5}$.

Meier et al. ${ }^{6}$ propuseram uma ruptura intencional das lesões intermediárias com a angioplastia com o balão no momento do cateterismo. A placa rica em lípides rompida tornar-se-ia fibrótica e o risco de evento coronário poderia ser eliminado. O procedimento foi chamado de "selamento da placa"7. Desde então, abordagens terapêuticas locais percutâneas inovadoras têm sido propostas para o tratamento de placas ateroscleróticas de alto risco.

Os stents não-farmacológicos (SNF) são utilizados para estabilizar placas rotas em pacientes sintomáticos e também podem estabilizar fibroateromas de capa fina (FACF) em pacientes assintomáticos, o tipo mais comum de placa aterosclerótica de alto risco ${ }^{8}$. Entretanto, esse efeito benéfico é obtido à custa do espessamento neo-intimal induzido pelo stent. Além disso, o próprio stent pode romper a capa fina e induzir uma resposta cicatricial mais agressiva ${ }^{9}$. Considerando o fato de terem potencial de reduzir o espessamento neo-intimal, os stents farmacológicos po- and associated with increased neointima in BMS but not in EES and BES.

DESCRIPTORS: Atherosclerosis. Stents. Coronary restenosis. Recurrence. Models, animal. Rabbits.

dem combinar os efeitos estabilizadores dos SNF com risco reduzido de reestenose. Estudos in vitro, experimentais e clínicos demonstraram reduções na reestenose com a utilização de stents liberadores de sirolimus ${ }^{10,11}$. O everolimus [40-O-(2-hidroxi-etil)-rapamicina], um composto ativo imunossupressor e antiproliferativo, da mesma família do sirolimus, mostrou-se promissor na prevenção de reestenose em modelos experimentais $^{12}$ e testes clínicos ${ }^{1-15}$ e poderia estabilizar os FACF.

Os efeitos antiproliferativos e antimitogênicos do estrogênio, mediados por vias regulatórias da transcrição de genes, serviram como base teórica para os stents liberadores de 17 - $\beta$-estradiol $(S L B)^{16}$. Dados experimentais utilizando SLB demonstraram redução da proliferação neo-intimal em comparação aos SNF ${ }^{17,18}$ e estes também poderiam estabilizar os FACF.

Este estudo teve por objetivo avaliar os efeitos estabilizadores dos SNF em comparação com os stents liberadores de everolimus (SLE) e os SLB em um modelo experimental de FACF em animais com aterosclerose crônica.

\section{MÉTODO}

\section{Modelo experimental}

O Institutional Animal Care and Use Committee, da Universidade de Kentucky, Estados Unidos, aprovou o protocolo. O estudo incluiu 16 coelhos brancos da raça Nova Zelândia, que foram colocados em uma dieta suplementada com $1 \%$ de colesterol (Purina Mills Inc. - Richmond, Indiana, Estados Unidos) por períodos de 2 meses, alternados com dieta normal, por um total de 8 meses e acompanhados por um período mínimo de $4 \operatorname{anos}^{19}$. As lesões ateroscleróticas observadas em 4 anos incluíram depósitos de cristal de colesterol, núcleo necrótico e formação e calcificação da capa fibrosa, de forma similar à observada na doença aterosclerótica avançada em humanos (Figura 1).

\section{Protocolo experimental}

Em 5 animais foram implantados SLE, outros 5 receberam SLB e os 6 remanescentes receberam SNF (Multilink Penta ${ }^{\mathrm{T}}$ ) (Advanced Cardiovascular Systems Santa Clara, Califórnia, Estados Unidos). No grupo SLE, foi utilizado um modelo com conteúdo de everolimus de $269 \mu \mathrm{g}$ por stent, com liberação de 15\% em 24 
Echeverri D, et al. Estabilização da Placa Aterosclerótica por Stents Farmacológicos e Não-Farmacológicos em Modelo Experimental de Fibroateroma de Capa Fina em Coelhos. Rev Bras Cardiol Invas. 2008;16(2):170-177.

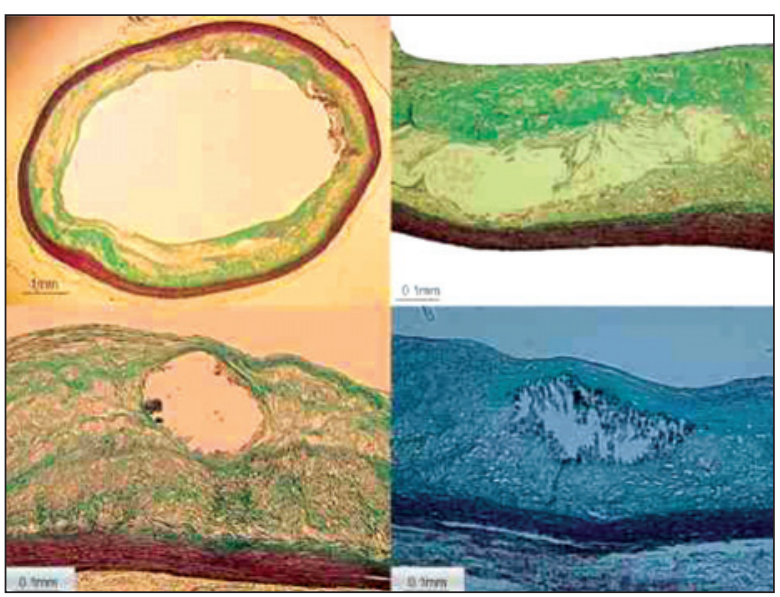

Figura 1 - Lesões ateroscleróticas avançadas em um modelo de coelho aterosclerótico senescente (4-5 anos). As imagens mostram os fibroateromas de capa fina com coloração pelo tricrômio para elastina, em que se observam as características da capa fibrosa, o núcleo lipídico e a deposição de cristais de colesterol.

horas e extensão de $8 \mathrm{~mm}$. No grupo SLB, o modelo tinha conteúdo de estradiol de 295 mg por stent, liberação de $29 \%$ em 24 horas e extensão de 8 mm. Esses stents tinham matriz de fluoropolímero projetada para liberar $80 \%$ dos fármacos nos primeiros 30 dias após o implante. Cada animal recebeu 4 stents seqüenciais na aorta abdominal. Além disso, 1 stent com polímero e 1 SNF foram implantados para comparação.

Todos os animais receberam $10 \mathrm{mg} / \mathrm{kg}$ de aspirina por via oral na noite anterior ao implante do stent. A indução de anestesia foi obtida com ketamina por via intravenosa $(20 \mathrm{mg} / \mathrm{kg})$ e xilazina $(4 \mathrm{mg} / \mathrm{kg})$ injetadas em veia marginal da orelha. A anestesia geral foi mantida com $2 \%$ de isoflourano e oxigênio após a entubação endotraqueal. A arteriotomia cirúrgica da artéria femoral comum direita com a posterior inserção de um introdutor 4F foi seguida de anticoagulação com 100 UI/kg de heparina intravenosa. Aortograma retrógrado distal foi realizado com injeção manual de contraste. Um fio de $0,014^{\prime \prime}$ foi introduzido sob fluoroscopia da artéria femoral até o segmento superior da aorta descendente, acima das artérias renais. Os stents foram implantados seqüencialmente no segmento distal da aorta abdominal, abaixo da artéria renal esquerda, com pressão de $14 \pm 2$ atm e razão diâmetro do stentdiâmetro da artéria de 1:1. Cuidado especial foi tomado para evitar o óstio das artérias lombares dentro dos segmentos cobertos pelos stents. Após o implante dos stents foi realizado aortograma para verificar se todos os stents foram implantados sem complicações. No final do procedimento, o introdutor foi removido e a artéria femoral foi ligada. Todos os animais foram observados durante a recuperação da anestesia e, então, devolvidos ao biotério. Após 28 dias de acompanhamento, foi realizada eutanásia eletiva com o uso de $150 \mathrm{mg} / \mathrm{kg}$ de pentobarbital intravenoso.

\section{Avaliação patológica}

Imediatamente após a eutanásia, a aorta proximal foi canulada para permitir sua fixação com a perfusão de formalina tamponada neutra a $10 \%$, com pressão de $60 \mathrm{mmHg}$ a $80 \mathrm{mmHg}$, por 30 minutos. O segmento aórtico abaixo da artéria renal esquerda foi dissecado cuidadosamente, incrustado em metilmetacrilato e, para as lesões que receberam stent (grupos experimentais), seccionado a cada 4-5 $\mu \mathrm{m}$ com lâmina de carboneto de aço inoxidável (Wasatch Histo Consultants - Winnemucca, Nevada, Estados Unidos). O segmento aórtico desde a aorta torácica até a aorta abdominal, acima da artéria renal esquerda (aorta sem stent), foi incrustado em parafina e seccionado para a obtenção das lesões de novo (grupo de controle). Foi feita a comparação entre 12 segmentos (3 segmentos por stent, 4 stents por animal) e 20 segmentos de novo (sem stents) por animal.

\section{Morfometria}

Definição de FACF: O FACF foi definido como uma placa com espessura mínima da capa fibrosa $\leq 65$ $\mu \mathrm{m}$ infiltrada com macrófagos ${ }^{20}$ e com área de núcleo de lipídio $\geq 30 \%$ da área total de placa (Figura 1).

Aposição do stent e quantificação do dano vascular: As hastes dos stents foram avaliadas para verificação de sua aposição por meio da análise histológica. O dano à parede do vaso também foi quantificado em cada haste, utilizando-se escore de 1 a $3^{21}$.

Análise da seção transversal: A placa foi centrada no meio do campo do microscópio, utilizando-se uma base mecânica deslizante manual calibrada. Com uma objetiva com aumento de $2 \mathrm{x}$, a área de placa, a área de lípide e a espessura da capa fibrosa foram quantificadas por planimetria computadorizada, utilizando o sistema Zedex, como relatado anteriormente ${ }^{22}$. Nas seções com stent, a área da placa foi calculada subtraindo-se a área do vaso (área da lâmina elástica interna) da área do $\operatorname{stent}^{23}$. A espessura da capa fibrosa foi definida como a espessura do conjunto colágeno, células musculares lisas e matriz extracelular que separava o núcleo lipídico do lúmen e foi quantificada por micrometria ocular. A Figura 2 exibe um exemplo de seção transversal de um stent estudado em análise do segmento. O número de hastes implantadas em segmentos arteriais normais ou doentes seguiu uma distribuição randômica e imprevisível. Além disso, o escore de danos à parede do vaso variou de 0 a 3 no mesmo segmento de seção transversal.

Análise haste por haste: Para comparação, somente as lesões de novo que atendiam à definição de FACF foram incluídas. A placa foi centrada no meio do campo do microscópio, utilizando uma base mecânica deslizante manual calibrada. Com uma objetiva com aumento de 20x, a área de acúmulo de lípide $\left(\mathrm{mm}^{2}\right)$ e a área da capa fibrosa $\left(\mathrm{mm}^{2}\right)$ foram quantificadas por 
Echeverri D, et al. Estabilização da Placa Aterosclerótica por Stents Farmacológicos e Não-Farmacológicos em Modelo Experimental de Fibroateroma de Capa Fina em Coelhos. Rev Bras Cardiol Invas. 2008;16(2):170-177.

planimetria computadorizada. Todas as seções com stent foram cuidadosamente analisadas para identificar hastes implantadas em lesões seguindo os mesmos critérios de seleção dos FACF. Desta vez, a haste foi centrada no meio do campo do microscópio. Com uma objetiva com aumento de 20x, a área de acúmulo de lípide $\left(\mathrm{mm}^{2}\right)$, a área da capa fibrosa antiga $\left(\mathrm{mm}^{2}\right)$ e a área da capa fibrosa nova, composta pelas áreas da capa fibrosa antiga e a área de proliferação neo-intimal $\left(\mathrm{mm}^{2}\right)$, foram quantificadas (Figura 3).

Ruptura da capa fibrosa com stent: A integridade/ ruptura da capa fibrosa após implante do stent foi registrada sistematicamente pela microscopia de luz, utilizando-se a objetiva com aumento de 20x. A proliferação neo-intimal e a cicatrização da parede do vaso

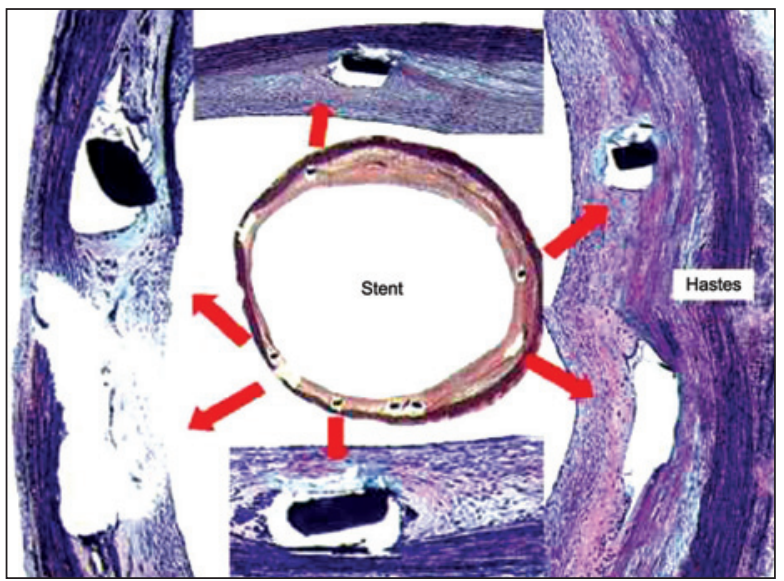

Figura 2 - Seção transversal do segmento do stent. Segmento da aorta no centro da figura (coloração pelo tricômio para elastina; campo 2x). Ao redor aparecem hastes com diferentes escores para dano vascular e proliferação neo-intimal. Notam-se os diferentes danos à placa e as diferentes respostas neo-intimais (setas vermelhas) (coloração pelo tricômio para elastina, campo 40x).

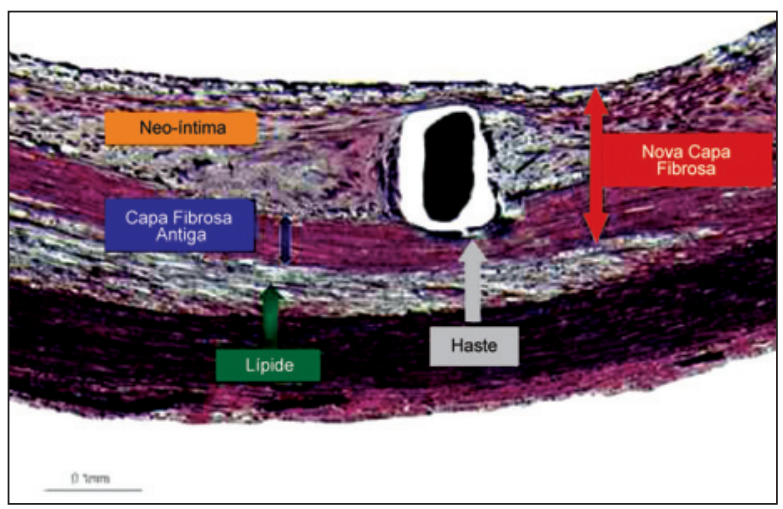

Figura 3 - Seção histológica mostrando diferentes estruturas do fibroateroma de capa fina após implante de stent. A haste está comprimindo a capa fibrosa antiga (seta cinza). A neo-íntima desenvolveu-se, espessando a nova capa fibrosa (seta vermelha). Seção processada pelo método de coloração do tricômio para elastina; campo 20x. foram quantificadas, assim como foi avaliada sua relação com a integridade da capa fibrosa.

\section{Análise estatística}

As variáveis contínuas foram apresentadas como média \pm erro padrão. Os dados morfométricos que seguiram distribuição não-gaussiana foram comparados por meio do teste não-paramétrico de MannWhitney. Variáveis que seguiram distribuição gaussiana foram comparadas com o teste $t$ de Student bicaudal. Variáveis dicotômicas foram comparadas com o teste do qui-quadrado. A significância foi estabelecida para um valor de $\mathrm{p} \leq 0,05$. O software Statistical Package for Social Science (SPSS) 10.0.5 foi utilizado para as análises.

\section{RESULTADOS}

Todos os 16 coelhos completaram o procedimento com sucesso sem complicações agudas. Três coeIhos morreram depois de 4, 8 e 23 dias do implante do stent. A necropsia identificou insuficiência renal aguda em 2 coelhos e grande infarto esplênico no terceiro coelho. Os 13 animais remanescentes foram submetidos a eutanásia eletiva 28 dia após o implante do stent. Dos 260 segmentos não tratados avaliados, 76 demonstraram placas ateroscleróticas e foram incluídos como grupo controle (de novo). Adicionalmente, dos 156 segmentos com stent avaliados, 138 demonstraram placas ateroscleróticas atrás das hastes e foram incluídos como grupo com stent.

Aposição de stent e escore de dano vascular: Dos 156 segmentos com stent, 1.584 hastes foram sistematicamente analisadas para aposição de stent. Ficaram apropriadamente apostas à parede do vaso 1.529 (98\%) hastes. $\mathrm{O}$ escore médio de dano à parede do vaso foi de $0,66 \pm 0,03$.

Análise da seção transversal por tipo de stent: $A$ análise da seção transversal mostrou diferentes morfologias de placa ao comparar segmentos de novo com segmentos com SNF e stents farmacológicos (Tabela 1). Em comparação com os segmentos de novo, os segmentos com SNF mostraram redução das áreas de placa e área de acúmulo de lípides, redução do porcentual de área com acúmulo de lípides e aumento da espessura da capa fibrosa ( $p \leq 0,0001$ para ambas as comparações). Os segmentos com SLE e SLB também mostraram porcentual de área de acúmulo de lípides reduzida e maior espessura de capa fibrosa ( $p \leq$ 0,05 para todas as comparações).

Análise haste por haste por tipo de stent: Dos 76 segmentos de novo, 33 placas ateroscleróticas atenderam ao critério histológico de FACF e foram incluídas como placas de novo. Das 1.584 hastes avaliadas, 237 foram implantadas em placas que atendiam aos critérios histológicos de FACF e foram incluídas como 
Echeverri D, et al. Estabilização da Placa Aterosclerótica por Stents Farmacológicos e Não-Farmacológicos em Modelo Experimental de Fibroateroma de Capa Fina em Coelhos. Rev Bras Cardiol Invas. 2008;16(2):170-177.

placas com stents. Os resultados planimétricos são apresentados na Tabela 2. Em comparação às placas de novo, todos os stents mostraram área de lípides reduzida, área de capa fibrosa antiga reduzida e área de capa fibrosa nova aumentada $(\mathrm{p}<0,0001$ para todas as comparações). Comparando SNF aos SLE e SLB, a área de lípide e a área da capa fibrosa antiga foram similares $(p=n s)$. Entretanto, a área de capa fibrosa nova foi reduzida significativamente pelos SLE $(1,05 \pm$ $0,4 \mathrm{~mm}^{2}$ vs. $\left.0,73 \pm 0,4 \mathrm{~mm}^{2} ; \mathrm{p}=0,0001\right)$, e reduzida marginalmente pelos SLB $\left(1,05 \pm 0,4 \mathrm{~mm}^{2}\right.$ vs. 0,88 \pm $\left.0,7 \mathrm{~mm}^{2} ; \mathrm{p}=0,05\right)$. As comparações entre SLE e SLB não mostraram diferenças significativas $(p=n s)$. A Figura 4 mostra exemplos de hastes de SNF, SLE e SLB implantadas em FACF.

Ruptura da capa fibrosa com stent: Das 237 hastes implantadas em FACF analisadas, 149 (63\%) mostraram ruptura da capa fibrosa induzida pela haste. $\mathrm{Na}$ análise de SNF, as áreas neo-intimais eram maiores em FACF com ruptura da capa fibrosa induzida pela haste em comparação aos FACF sem ruptura da capa fibrosa $\left(0,85 \pm 0,05 \mathrm{~mm}^{2}\right.$ vs. 0,66 $\pm 0,06 \mathrm{~mm}^{2}$; $\mathrm{p}=0,03)$, como mostrado na Figura 5. Na análise do $\operatorname{SLE}\left(0,53 \pm 0,05 \mathrm{~mm}^{2}\right.$ vs. 0,45 $\left.\pm 0,06 \mathrm{~mm}^{2} ; \mathrm{p}=0,35\right)$ e do $\operatorname{SLB}\left(0,71 \pm 0,08 \mathrm{~mm}^{2}\right.$ vs. 0,53 $\pm 0,11 \mathrm{~mm}^{2} ; p$ $=0,19)$, as áreas neo-intimais foram similares aos FACF, com ou sem ruptura da capa fibrosa induzida pelas hastes.

\section{DISCUSSÃO}

Este estudo avaliou pela primeira vez as respostas biológicas dos FACF submetidos ao implante de stent em um modelo de aterosclerose crônica experimental em coelhos. Para obter lesões ateroscleróticas comparáveis às dos humanos adultos, o modelo animal foi exposto a dieta de colesterol mais elevado que o usual e por um período mais longo (intermitentemente por 8 meses ao invés de 16 semanas) e acompanhado por pelo menos 4 anos, após completar o protocolo da dieta. Os coelhos com mais de 4 anos de idade são considerados senis e notamos o desenvolvimento de aterosclerose avançada até então desconhecida para esse modelo de animal. As placas aórticas tinham capas fibrosas finas, grandes núcleos necróticos com depósito de cristal de colesterol, infiltração de macrófagos e mesmo hemorragia intraplaca. Além disso, calcificação, uma característica de doença crônica humana, foi também freqüentemente observada. O desenvolvimento de um modelo animal com padrões histológicos similares aos da doença humana representa importante passo no estudo das placas vulneráveis. Além disso, esse modelo permitiu a cuidadosa avaliação da microarquitetura do FACF com ou sem os diferentes tipos de stents implantados, incluindo SNF, SLE e SLB.

O segundo resultado importante deste estudo foi a abordagem mecanicista combinada para avaliar a estabilização induzida pelos stents em FACF. As análi-

TABELA 1

Análise de segmentos de novo comparados aos segmentos com stent (stents farmacológicos e stents não-farmacológicos)

\begin{tabular}{lcccc} 
& Lesões de novo & SNF & SLB & SLE \\
& $\mathbf{n}=\mathbf{7 6}$ & $\mathbf{n}=\mathbf{6 5}$ & $\mathbf{n = 2 0}$ & $\mathbf{n}=\mathbf{2 2}$ \\
\hline Área da placa $\left(\mathrm{mm}^{2}\right)$ & $2,8 \pm 0,19$ & $1,75 \pm 0,13^{*}$ & $2,56 \pm 0,27^{*}$ & $1,9 \pm 0,15^{*}$ \\
Área de lípide $\left(\mathrm{mm}^{2}\right)$ & $1,43 \pm 0,1$ & $0,63 \pm 0,05^{*}$ & $0,61 \pm 0,1^{*}$ & $0,21 \pm 0,04^{*}$ \\
Área de lípide $(\%)$ & $47,7 \pm 1,2$ & $34 \pm 1,1^{*}$ & $22 \pm 3^{*}$ & $11 \pm 2^{*}$ \\
Espessura mínima da capa $(\mathrm{mc})$ & $23 \pm 2$ & $116 \pm 8,0^{*}$ & $31 \pm 5^{*}$ & $59 \pm 13^{*}$ \\
\hline
\end{tabular}

* $\mathrm{p}<0$ 0,05 em comparação com grupo de novo; $\mathrm{p}<0,05$ em comparação com stent não-farmacológico.

SLB = stent com liberação de 17- $\beta$ estradiol; SLE = stent com liberação de everolimus; SNF = stent não-farmacológico.

TABELA 2

Análise haste por haste por tipo de stent

\begin{tabular}{lcccc}
\hline Análise haste por haste & $\begin{array}{c}\text { SNF } \\
\mathbf{n = 1 2 7}\end{array}$ & $\begin{array}{c}\text { Polímero } \\
\mathbf{n}=\mathbf{2 3}\end{array}$ & $\begin{array}{c}\text { SLB } \\
\mathbf{n}=\mathbf{4 6}\end{array}$ & $\begin{array}{c}\text { SLE } \\
\mathbf{n}=\mathbf{4 1}\end{array}$ \\
\hline Área de lípide $\left(\mathrm{mm}^{2}\right)$ & $0,39 \pm 0,2$ & $0,39 \pm 0,5$ & $0,39 \pm 0,4$ & $0,37 \pm 0,3$ \\
Área de capa fibrosa antiga $\left(\mathrm{mm}^{2}\right)$ & $0,26 \pm 0,1$ & $0,37 \pm 0,4^{*}$ & $0,24 \pm 0,2$ & $0,23 \pm 0,2$ \\
Área de capa fibrosa nova $\left(\mathrm{mm}^{2}\right)$ & $1,05 \pm 0,4$ & $1,1 \pm 0,6$ & $0,88 \pm 0,7^{*}$ & $0,73 \pm 0,4^{*}$ \\
\hline
\end{tabular}

$* p<0,05$ em comparação com o grupo stent não-farmacológico; $p<0,05$ em comparação com o grupo de polímero.

SLB = stent com liberação de 17-b estradiol; SLE = stent com liberação de everolimus; SNF = stent não-farmacológico. 
Echeverri D, et al. Estabilização da Placa Aterosclerótica por Stents Farmacológicos e Não-Farmacológicos em Modelo Experimental de Fibroateroma de Capa Fina em Coelhos. Rev Bras Cardiol Invas. 2008;16(2):170-177.

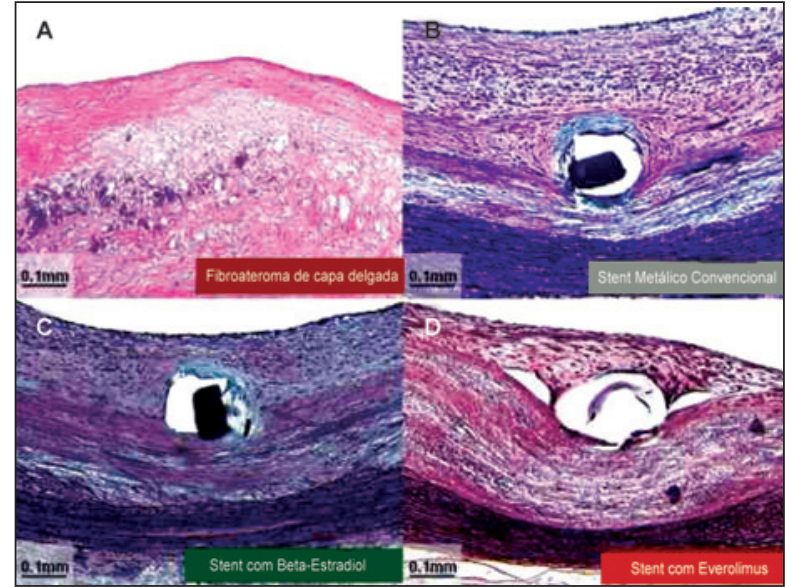

Figura 4 - Figura exibindo exemplos de seções de novo (sem stent) e com stent. A. Fibroateromas de capa fina de novo com depósitos calcificados (coloração H\&E; campo 20x). B. Seção de stent nãofarmacológico, mostrando espessamento neo-intimal intenso (coloração pelo tricômio para elastina; campo 20x). C. Seção de stent com liberação de 17- $\beta$ estradiol. D. Seção de stent com liberação de everolimus, mostrando menor espessamento neo-intimal (coloração pelo tricômio para elastina; campo 20x).

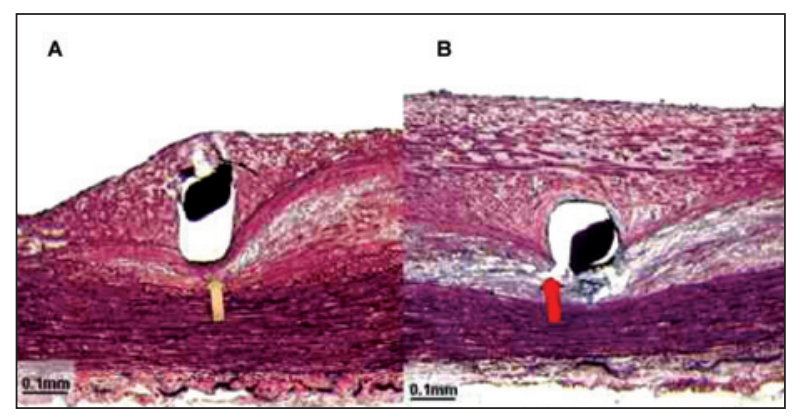

Figura 5 - Relação entre haste e capa fibrosa. O painel A mostra capa fibrosa intacta (escore de dano à placa aterosclerótica: 0). O painel B mostra a ruptura da capa fibrosa (escore de dano à placa aterosclerótica: 1) Coloração pelo tricômio para elastina; campo 20x.

ses de seção transversal demonstraram reduções significativas do núcleo lipídico com aumento da espessura da capa fibrosa nos segmentos tratados com stents em comparação com os segmentos de novo. As análises de seção transversal são atualmente o método recomendado para avaliar os danos à parede do vaso após o implante de stents farmacológicos ${ }^{23}$. Entretanto, a avaliação de FACF por esse método pode ser influenciada pela aposição aleatória das hastes do stent, tanto na íntima normal como na doente, com inúmeros escores de dano vascular, imprevisíveis e muito variáveis, dentro do mesmo segmento de seção transversal. Conseqüentemente, o questionamento específico a respeito da estabilização dos FACF com implante de stents farmacológicos e SNF requereu análise adicional, haste por haste, mais detalhada e sistemática. Utilizando método adaptado de Farb et al. ${ }^{9}$, identi- ficamos reduções na área de acúmulo de lípides, juntamente com aumentos significativos na área de capa fibrosa dos FACF tratados com stents em comparação aos FACF não tratados. Os stents farmacológicos, especificamente os SLE, demonstraram espessamento da capa fibrosa mais controlado, com potenciais implicações clínicas no que diz respeito a menores taxas de reestenose. Note-se que as áreas de capa fibrosa antiga foram reduzidas por SNF e stents farmacológicos, refletindo perda de tecido após a ruptura da capa fibrosa induzida pela haste do stent. Como resultado, dois métodos diferentes, a análise de seção transversal e a análise haste por haste, demonstraram de maneira consistente padrões de estabilização dos FACF com stents farmacológicos e SNF em comparação às lesões de FACF de novo não tratadas com stents. As áreas de acúmulo lipídico também foram reduzidas em todas as seções com stent. Os mecanismos envolvidos nessa observação são incertos, mas podem estar relacionados às forças de compressão aplicadas durante o implante do stent com inibição adicional da retração elástica do vaso.

A terceira conclusão relevante está relacionada à ruptura da capa fibrosa induzida por haste, achado freqüente com a utilização da atual tecnologia de implante de stent. Cerca de dois terços das hastes romperam a capa fibrosa quando implantadas nessas frágeis placas dos FACF, mesmo com o baixo escore de dano à parede do vaso encontrado $(0,66 \pm 0,03)$. Esse resultado surpreendente ressalta a importância de um planejamento metodológico para avaliar questões específicas em pesquisa experimental. Um escore desenvolvido para vasos saudáveis ${ }^{21}$ pode ser limitado em vasos com doença crônica avançada. O mais importante é que a ruptura da capa fibrosa induzida pelos SNF foi associada a aumento significativo na área neo-íntima, proporcionando uma perspectiva clinicamente relevante para o dano mecânico quando do implante do stent no FACF. Esses resultados estão de acordo com achados anatomopatológicos em humanos, que demonstram que a ruptura da capa fibrosa induzida pela haste está associada a aumento da espessura neo-intimal, infiltrado inflamatório e formação vascular neo-intimal ${ }^{9}$. Note-se que essa resposta neo-intimal agressiva após a ruptura da capa fibrosa induzida pela haste pode ser modulada tanto pelo SLE como pelo SLB.

Depois da importante observação de que os maiores diâmetros luminais obtidos com o implante de stent de alta pressão estão associados a menor trombose aguda e subaguda e a menores taxas de reestenose ${ }^{24,25}$, o implante de stents com alta pressão tem sido a norma para o tratamento percutâneo das lesões coronárias. O cardiologista intervencionista adapta o stent à doença e não a doença ao stent. Entretanto, a atual tecnologia de implante dos stents não permite a quantificação dos danos à placa induzida pelas hastes. Como resultado, essa abordagem universal do implante com alta pressão necessita reanálise cuidadosa quando 
Echeverri D, et al. Estabilização da Placa Aterosclerótica por Stents Farmacológicos e Não-Farmacológicos em Modelo Experimental de Fibroateroma de Capa Fina em Coelhos. Rev Bras Cardiol Invas. 2008;16(2):170-177.

se planeja tratar FACF. Basicamente, a consideração em primeiro lugar da doença e depois do stent a ser utilizado pode ter valor significativo para a estabilização da placa. Entretanto, estudos adicionais são necessários para elucidar totalmente essa complexa questão.

\section{Limitações}

O período de 28 dias de acompanhamento não é suficiente para a avaliação apropriada dos stents farmacológicos. Além disso, reduções da espessura da nova capa fibrosa observadas com os stents farmacológicos podem refletir apenas atrasos no espessamento neo-intimal que poderiam desaparecer em acompanhamento prolongado. Como resultado, estudos com seguimento tardio estão sendo desenhados para confirmar essas observações.

\section{CONCLUSÃO}

O implante de stent em FACF aumenta a espessura da capa fibrosa e reduz o conteúdo de lípides em modelo experimental de aterosclerose avançada. Além disso, os SLE e os SLB estão associados a resposta neo-intimal controlada. Finalmente, a ruptura da capa fibrosa é achado comum no implante de stent em FACF. Esse evento relacionado ao procedimento está associado a maior espessamento neo-intimal quando o stent não-farmacológico é utilizado e pode ser reduzido pela utilização de stents farmacológicos. As técnicas atuais de implante de stent de alta pressão necessitam de cuidadosa reconsideração no implante de stent em FACF.

\section{AGRADECIMENTOS}

Os autores agradecem aos drs. Randy Stevens, Ken Dickey, Mark Mathew e Gentian Kristo por sua ajuda durante os procedimentos experimentais, e ao sr. Terry Lacy por sua expertise em histotecnologia. Os autores também expressam seu reconhecimento à Equipe de Placa Vulnerável da Guidant Corporation, pelo patrocínio e apoio diligente em todas as fases do estudo.

\section{REFERÊNCIAS BIBLIOGRÁFICAS}

1. Buffon A, Biasucci LM, Liuzzo G, D'Onofrio G, Crea F, Maseri A. Widespread coronary inflammation in unstable angina. N Engl J Med. 2002;347(1):5-12.

2. Rioufol G, Finet G, Ginon I, André-Fouët X, Rossi R, Vialle $E$, et al. Multiple atherosclerotic plaque rupture in acute coronary syndrome: a three-vessel intravascular ultrasound study. Circulation. 2002;106(7):804-8.

3. Ridker PM, Cannon CP, Morrow D, Rifai N, Rose LM, $\mathrm{McC}$ abe $\mathrm{CH}$, et al. Pravastatin or Atorvastatin Evaluation and Infection Therapy-Thrombolysis in Myocardial Infarction 22 (PROVE IT-TIMI 22) Investigators. C-reactive protein levels and outcomes after statin therapy. $N$ Engl J Med. 2005;352(1):20-8.

4. Goldstein JA, Demetriou D, Grines CL, Pica M, Shoukfeh $\mathrm{M}, \mathrm{O}^{\prime}$ Neill WW. Multiple complex coronary plaques in patients with acute myocardial infarction. $N$ Engl J Med. 2000;343(13):915-22.
5. Keeley EC, Boura JA, Grines CL. Primary angioplasty versus intravenous thrombolytic therapy for acute myocardial infarction: a quantitative review of 23 randomised trials. Lancet. 2003;361(9351):13-20.

6. Meier B, Ramamurthy S. Plaque sealing by coronary angioplasty. Cathet Cardiovasc Diagn. 1995;36(4):295-7.

7. Meier B. Plaque sealing by coronary angioplasty. Heart. 2004;90(12):1395-8.

8. Kolodgie FD, Burke AP, Farb A, Gold HK, Yuan J, Narula J, et al. The thin-cap fibroatheroma: a type of vulnerable plaque: the major precursor lesion to acute coronary syndromes. Curr Opin Cardiol. 2001;16(5):285-92.

9. Farb A, Weber DK, Kolodgie FD, Burke AP, Virmani R. Morphological predictors of restenosis after coronary stenting in humans. Circulation. 2002;105(25):2974-80.

10. Gallo R, Padurean A, Jayaraman T, Marx S, Roque M, Adelman $\mathrm{S}$, et al. Inhibition of intimal thickening after balloon angioplasty in porcine coronary arteries by targeting regulators of the cell cycle. Circulation. 1999;99(16):2164-70.

11. Sousa JE, Costa MA, Sousa AG, Abizaid A, Seixas AC, Abizaid AS, et al. Two-year angiographic and intravascular ultrasound follow-up after implantation of sirolimus-eluting stents in human coronary arteries. Circulation. 2003;107(3): 381-3.

12. Farb A, John M, Acampado E, Kolodgie FD, Prescott MF, Virmani R. Oral everolimus inhibits in-stent neointimal growth. Circulation. 2002;106(18):2379-84.

13. Costa RA, Lansky AJ, Mintz GS, Mehran R, Tsuchiya $Y$ Negoita $M$, et al. Angiographic results of the first human experience with everolimus-eluting stents for the treatment of coronary lesions (the FUTURE I trial). Am J Cardiol. 2005;95(1):113-6.

14. Serruys PW, Ong ATL, Piek JJ, Neumann F, van der Giessen WJ, Wiemer $\mathrm{M}$, et al. A randomized comparison for a durable polymer everolimus eluting stent with a bare metal coronary stent: The SPIRIT first trial. Eurointerv. 2005;1:58-65.

15. Serruys PW, Ruygrok P, Neuzner J, Piek JJ, Seth A, Schofer $\mathrm{JJ}$, et al. A randomized comparison of an everolimus-eluting coronary stent with a paclitaxel-eluting coronary stent: The SPIRIT II trial. Eurointerv. 2006;2:286-94.

16. Nadal A, Díaz M, Valverde MA. The estrogen trinity: membrane, cytosolic, and nuclear effects. News Physiol Sci. 2001;16:2515.

17. Li G, Chen YF, Greene GL, Oparil S, Thompson JA. Estrogen inhibits vascular smooth muscle cell-dependent adventitial fibroblast migration in vitro. Circulation. 1999; 100(15):1639-45.

18. New G, Moses JW, Roubin GS, Leon MB, Colombo A, Iye SS, et al. Estrogen-eluting, phosphorylcholine-coated stent implantation is associated with reduced neointimal formation but no delay in vascular repair in a porcine coronary model. Catheter Cardiovasc Interv. 2002;57(2):266-71.

19. Olivetti G, Melissari M, Capasso JM, Anversa P. Cardiomyopathy of the aging human heart. Myocyte loss and reactive cellular hypertrophy. Circ Res. 1991;8(6):1560-8.

20. Burke AP, Farb A, Malcom GT, Liang YH, Smialek J, Virmani R. Coronary risk factors and plaque morphology in men with coronary disease who died suddenly. N Engl J Med. 1997;336(18):1276-82

21. Schwartz RS, Huber KC, Murphy JG, Edwards WD, Camrud $A R$, Vlietstra RE, et al. Restenosis and the proportional neointimal response to coronary artery injury: results in a porcine model. J Am Coll Cardiol. 1992;19(2):267-74.

22. Moreno PR, Lodder RA, Purushothaman KR, Charash WE, $\mathrm{O}^{\prime}$ Connor WN, Muller JE. Detection of lipid pool, thin fibrous cap, and inflammatory cells in human aortic atheroscle- 
Echeverri D, et al. Estabilização da Placa Aterosclerótica por Stents Farmacológicos e Não-Farmacológicos em Modelo Experimental de Fibroateroma de Capa Fina em Coelhos. Rev Bras Cardiol Invas. 2008;16(2):170-177.

rotic plaques by near-infrared spectroscopy. Circulation. 2002;105(8):923-7.

23. Schwartz RS, Edelman ER, Carter A, Chronos N, Rogers C, Robinson KA, et al. Drug-eluting stents in preclinical studies: recommended evaluation from a consensus group. Circulation. 2002;106(14):1867-73.

24. Kuntz RE, Safian RD, Carrozza JP, Fishman RF, Mansour M,
Baim DS. The importance of acute luminal diameter in determining restenosis after coronary atherectomy or stenting. Circulation. 1992;86(6):1827-35.

25. Colombo A, Hall P, Nakamura S, Almagor Y, Maiello L, Martini G, et al. Intracoronary stenting without anticoagulation accomplished with intravascular ultrasound guidance. Circulation. 1995;91(6):1676-88. 